\title{
ON THE $k$-TH POWER OF STOKES OPERATOR
}

\section{ELEFTHERIOS PROTOPAPAS}

Department of Mathematics

School of Applied Mathematical and Physical Sciences

National Technical University of Athens

Greece

e-mail: lprotopapas@math.ntua.gr

\begin{abstract}
Stokes operators, $E^{2}, E^{4}$, are well known partial differential operators of elliptic type, which are often used in Applied Mathematics. Stokes equation $E^{2} \psi=0$ describes the irrotational, axisymmetric creeping flow and Stokes bi-stream equation $E^{4} \psi=0$ denotes the rotational one, where $E^{4}=E^{2} \circ E^{2}$. Necessary and sufficient conditions for the separability and the R-separability of the equation $E^{2} \psi=0$ have been proved recently. Moreover, the 0-eigenspace and the generalized 0-eigenspace of the operator $E^{2}$ have been derived in several coordinate systems. Specifically, the spherical coordinate system is employed in many problems taking into account that in many engineering applications, the solutions in spherical geometry seem to be adequate for solving a problem. In the present manuscript, it is shown that equation $E^{2 k} \psi=0, k \in \mathbb{N}^{*}$ admits a solution of the form $U=u_{1}+r^{2} u_{2}+r^{4} u_{3}+\ldots+$ $r^{2 k-2} u_{k}$, where $u_{i}, i=1,2, \ldots, k$ are solutions of Stokes equation and $r$ is the radial spherical variable. Additionally, we obtain the kernel of the $k$-th power of the Stokes operator, $E^{2 k}$, in the spherical geometry for every $k \in \mathbb{N}^{*}$.
\end{abstract}

2010 Mathematics Subject Classification: 35J30, 35C05.

Keywords and phrases: Stokes operator, eigenfunctions, spherical system of coordinates, Gegenbauer functions.

Received June 26, 2020; Revised July 2, 2020

(C) 2020 Scientific Advances Publishers 


\section{Introduction}

The Laplacian, $\Delta=\nabla^{2}$, is the most common elliptic operator [1] that occurs in many physical phenomena, such as electric and gravitational potentials, the diffusion equation, wave propagation etc. The solution of each one of the equations $\Delta u=0, \Delta^{2} u=0$ has been derived in several coordinate systems [1], [2]. Moreover, detailed description of the conditions needed to be satisfied for Laplace's equation to be separable or $\mathrm{R}$-separable were first given by Eisenhart [3]. Moon and Spencer in a series of celebrated papers [4]-[8] expanded and completed Eisenhart's work. Furthermore, Almansi [9] proved that if $u_{1}, u_{2}$ are solutions of $\Delta u=0$, the solution of $\Delta^{2} U=0$ is $U=u_{1}+r^{2} u_{2}$, where $r$ is the Euclidean distance in the appropriate geometry. In this elaborated paper [9], he also derived the general solution of the equation $\Delta^{2 n} u=0, n \in \mathbb{N}^{*}$.

Stokes operator, $E^{2}$, is an elliptic operator used in the case of an irrotational axisymmetric creeping flow [10]. Stokes equation $E^{2} \psi=0$ separates variables in spherical [10], in paraboloid [11] and in spheroid coordinate systems [12], while it R-separates variables in inverted prolate spheroid [13], [14], in inverted oblate spheroid [15], in bispherical, in toroidal [16], in cardiod and in tangent sphere [11] coordinate systems. Recently, Hadjinicolaou and Protopapas [17] stated and proved the necessary and sufficient conditions that must be fulfilled when we seek for the simple or the R-separability of equation $E^{2} \psi=0$ in every axisymmetric geometry, employing the metric coefficients of the coordinate system.

The steady axisymmetric creeping flow is fully described by the stream function $\psi$, which satisfies the fourth order partial differential equation of elliptic type $E^{4} \psi=0$, where $E^{4}=E^{2} \circ E^{2}$ is the Stokes bi-stream operator [10]. The solution of the equation $E^{4} \psi=0$ is obtained by calculating the generalized eigenfunctions of Stokes equation. In the 
spherical geometry it separates variables [10], in the prolate and oblate spheroid it semiseparates variables [12] and in the inverted spheroid coordinate systems it $R$-semiseparates variables [13]-[15].

Charalambopoulos and Dassios [18] expanded the Almansi type approach for the bi-harmonic Laplace operator to bi-stream Stokes operator, stating that the knowledge of two arbitrary solutions $\psi_{1}, \psi_{2}$ of $E^{2} \psi=0$, provide the solution $\Psi=\psi_{1}+r^{2} \psi_{2}$ of $E^{4} \Psi=0$, where $r$ is the Euclidean distance of the coordinate system. Dassios and Vafeas [19] derived the Almansi type generalized eigenfunction in prolate coordinate system. The Almansi type approach for the solution of $E^{4} \psi=0$ provide a safe road map for its general solution in every axisymmetric coordinate system when the general solution of $E^{2} \psi=0$ is known.

In this paper, we provide the analogous to the Almansi [9] expression for the solution of the equation $E^{2 k} \psi=0$ proving that we only need to know the eigenfunctions of the Stokes operator in order to calculate the 0-eigenspace of $E^{2 k}=\underbrace{E^{2} \circ E^{2} \circ \ldots \circ E^{2}}_{k \text {-times }}$ for every $k \in \mathbb{N}^{*}$. Moreover, we derive the 0 -eigenspace of the operator $E^{2 k}$ for every $k \in \mathbb{N}^{*}$ in the spherical coordinate system.

The manuscript is organized as follows. In Section 2, we present Stokes operator, while in Section 3, we show a solution for the equation $E^{2 k} \psi=0, k \in \mathbb{N}^{*}$. In Section 4 , we derive the kernel of the $k$-th power of the Stokes operator in spherical geometry. Finally, in Section 5, we summarize the results of the present work. 


\section{Stokes Operator}

In every curvilinear axisymmetric system of coordinates $\left(q_{1}, q_{2}, \phi\right)$ the Stokes operator [10] is

$$
E^{2}=\varpi h_{1} h_{2}\left[\frac{\partial}{\partial q_{1}}\left(\frac{h_{1}}{\varpi h_{2}} \frac{\partial}{\partial q_{1}}\right)+\frac{\partial}{\partial q_{2}}\left(\frac{h_{2}}{\varpi h_{1}} \frac{\partial}{\partial q_{2}}\right)\right],
$$

where $\Phi$ is the radial cylindrical coordinate and $h_{1}, h_{2}$ are the metric coefficients of the system.

Using the spherical system of coordinates $(r, \theta, \phi)$ every point $\left(x_{1}, x_{2}, x_{3}\right)$ is described [1] with

$$
\left(x_{1}, x_{2}, x_{3}\right)=(r \sin \theta \cos \phi, r \sin \theta \sin \phi, r \cos \theta),
$$

where $r>0, \theta \in[0, \pi], \phi \in[0,2 \pi)$, we derive

$$
h_{1}=1, h_{2}=\frac{1}{r}, \Phi=r \sin \theta \text {, }
$$

so Stokes operator assumes the form

$$
E^{2}=\frac{\partial^{2}}{\partial r^{2}}-\frac{1}{r^{2}} \frac{\cos \theta}{\sin \theta} \frac{\partial}{\partial \theta}+\frac{1}{r^{2}} \frac{\partial^{2}}{\partial \theta^{2}}
$$

If we substitute $\zeta=\cos \theta \in[-1,1]$, we reach at

$$
E^{2}=\frac{\partial^{2}}{\partial r^{2}}+\frac{1-\zeta^{2}}{r^{2}} \frac{\partial^{2}}{\partial \zeta^{2}}
$$

\section{Solution for Equation $E^{2 k} \psi=0, k \in \mathbb{N}^{*}$}

Lemma 1. If $u$ is a solution of $E^{2 k} \psi=0, k \geq 2$, then $r^{2} u$ is a solution of $E^{2 k+2} \psi=0$, where $r$ is the Euclidean distance, $E^{2}$ is the Stokes operator and $E^{2 k+2}=E^{2 k} \circ E^{2}$. 
Proof.

$$
\begin{aligned}
E^{2 k+2}\left(r^{2} u\right) & =E^{2 k}\left(E^{2}\left(r^{2} u\right)\right) \\
& =E^{2 k}\left(2 u+4 r \frac{\partial u}{\partial r}\right) \\
& =E^{2 k}\left(4 r \frac{\partial u}{\partial r}\right) \\
& =E^{2 k-2}\left(E^{2}\left(4 r \frac{\partial u}{\partial r}\right)\right)=0 .
\end{aligned}
$$

Lemma 2. If $u_{1}, u_{2}, \ldots, u_{k}, k \geq 1$ belong to the 0-eigenspace of $E^{2}$, then

$$
U=u_{1}+r^{2} u_{2}+r^{4} u_{3}+\ldots+r^{2 k-2} u_{k}
$$

belongs to the 0-eigenspace of $E^{2 k}, k \in \mathbb{N}^{*}$.

Proof. • For $k=1$ the lemma holds true.

- For $k \geq 2$ since $u_{i} \in \operatorname{ker}\left(E^{2}\right), \forall i=1,2, \ldots, k, k \geq 2$, from Lemma 1 and for $i=1,2, \ldots, k$ it holds that

$$
\begin{aligned}
& -r^{2} u_{i} \in \operatorname{ker}\left(E^{4}\right), \\
& -r^{4} u_{i}=r^{2}\left(r^{2} u_{i}\right) \in \operatorname{ker}\left(E^{6}\right), \\
& -r^{6} u_{i}=r^{2}\left(r^{4} u_{i}\right) \in \operatorname{ker}\left(E^{8}\right), \\
& -\ldots \\
& -r^{2 k-2} u_{i}=r^{2}\left(r^{2 k-4} u_{i}\right) \in \operatorname{ker}\left(E^{2 k}\right) .
\end{aligned}
$$

Furthermore $\operatorname{ker}\left(E^{2}\right) \subset \operatorname{ker}\left(E^{4}\right) \subset \ldots \subset \operatorname{ker}\left(E^{2 k}\right)$, so $U \in \operatorname{ker}\left(E^{2 k}\right)$. 
From Lemma 2, we conclude that function $U$ defined in (6) is solution of $E^{2 k} \psi=0, k \in \mathbb{N}^{*}$.

\section{The Kernel of $E^{2 k}, k \in \mathbb{N}^{*}$ in Spherical}

\section{Coordinate System}

It has been proved [10] that the eigenfunctions of Stokes operator $E^{2}$ in the spherical system are

$$
\begin{gathered}
L_{n, 1}^{(1)}(r, \zeta)=r^{n} G_{n}(\zeta), \\
L_{n, 1}^{(2)}(r, \zeta)=r^{n} H_{n}(\zeta), \\
L_{n, 1}^{(3)}(r, \zeta)=r^{1-n} G_{n}(\zeta), \\
L_{n, 1}^{(4)}(r, \zeta)=r^{1-n} H_{n}(\zeta),
\end{gathered}
$$

for $n=0,1,2, \ldots$, where $G_{n}, H_{n}$ are Gegenbauer functions [20] of the first and the second kind, respectively.

Furthermore, the generalized eigenfunctions of Stokes operator $E^{2}$ in the spherical system [10] are

$$
\begin{aligned}
& L_{n, 2}^{(1)}(r, \zeta)=r^{n+2} G_{n}(\zeta), \\
& L_{n, 2}^{(2)}(r, \zeta)=r^{n+2} H_{n}(\zeta), \\
& L_{n, 2}^{(3)}(r, \zeta)=r^{3-n} G_{n}(\zeta), \\
& L_{n, 2}^{(4)}(r, \zeta)=r^{3-n} H_{n}(\zeta),
\end{aligned}
$$

for $n=0,1,2, \ldots$. 
Therefore, the zero-eigenspace of the Stokes bi-stream operator $E^{4}$ consists of all the functions $L_{n, m}^{(i)}, n \in \mathbb{N}^{*}, m=1,2, i=1,2,3,4$.

Theorem 1. In the spherical coordinate system $(r, \zeta, \phi)$ the functions

$$
\begin{aligned}
& L_{n, m}^{(1)}(r, \zeta)=r^{n+2 m-2} G_{n}(\zeta), \forall m=1,2, \ldots, k, \\
& L_{n, m}^{(2)}(r, \zeta)=r^{n+2 m-2} H_{n}(\zeta), \forall m=1,2, \ldots, k, \\
& L_{n, m}^{(3)}(r, \zeta)=r^{2 m-1-n} G_{n}(\zeta), \forall m=1,2, \ldots, k, \\
& L_{n, m}^{(4)}(r, \zeta)=r^{2 m-1-n} H_{n}(\zeta), \forall m=1,2, \ldots, k,
\end{aligned}
$$

for $n=0,1,2, \ldots$ belong to the kernel of the operator $E^{2 k}, k=1,2,3,4, \ldots$, where $E^{2 k}=\underbrace{E^{2} \circ E^{2} \circ \ldots \circ E^{2}}_{k \text {-times }}$ and $G_{n}, H_{n}$ are Gegenbauer functions.

Proof. If $k=1$ from (7) to (10), we easily verify that the functions $L_{n, 1}^{(i)}$ belong to the zero-eigenspace of $E^{2}$ [10].

- Assuming that $L_{n, k}^{(i)} \in \operatorname{ker}\left(E^{2 k}\right)$ we will prove that $L_{n, k+1}^{(i)} \in \operatorname{ker}\left(E^{2 k+2}\right)$, where

$$
\begin{gathered}
L_{n, k+1}^{(1)}(r, \zeta)=r^{n+2 k} G_{n}(\zeta), \\
L_{n, k+1}^{(2)}(r, \zeta)=r^{n+2 k} H_{n}(\zeta), \\
L_{n, k+1}^{(3)}(r, \zeta)=r^{2 k+1-n} G_{n}(\zeta), \\
L_{n, k+1}^{(4)}(r, \zeta)=r^{2 k+1-n} H_{n}(\zeta) .
\end{gathered}
$$

We have

$$
E^{2 k}\left(L_{n, k}^{(1)}(r, \zeta)\right)=E^{2 k}\left(r^{n+2 k-2} G_{n}(\zeta)\right)=0
$$


and

$$
E^{2 k+2}\left(L_{n, k+1}^{(1)}(r, \zeta)\right)=E^{2 k}\left(E^{2}\left(r^{n+2 k} G_{n}(\zeta)\right)\right) .
$$

Substituting (5) in (24) we derive

$$
E^{2 k+2}\left(L_{n, k+1}^{(1)}(r, \zeta)\right)=E^{2 k}((n+2 k)(n+2 k-1)-n(n-1)) r^{n+2 k-2} G_{n}(\zeta),
$$

and since $G_{n}(\zeta)$ satisfies the Gegenbauer equation

$$
\left(1-\zeta^{2}\right) \frac{d^{2} f(\zeta)}{d \zeta^{2}}+n(n-1) f(\zeta)=0
$$

we derive that

$$
E^{2 k+2} L_{n, k+1}^{(1)}(r, \zeta)=2 k(2 k+2 n-1) E^{2 k}\left(r^{n+2 k-2} G_{n}(\zeta)\right)=0 .
$$

The proof is similar for the functions $L_{n, k+1}^{(i)}(r, \zeta), i=2,3,4$.

Lemma 3. In the spherical coordinate system $(r, \zeta)$ the solution of the equation $E^{2 k} \psi(r, \zeta)=0, k=1,2,3,4, \ldots$ is

$$
\begin{aligned}
\psi_{2 k}(r, \zeta)= & \sum_{n=0}^{+\infty}\left(\sum_{\nu=0}^{k-1} A_{n, 2 \nu} r^{n+2 \nu}\right) G_{n}(\zeta)+\sum_{n=0}^{+\infty}\left(\sum_{\nu=0}^{k-1} B_{n, 2 \nu} r^{n+2 \nu}\right) H_{n}(\zeta) \\
& +\sum_{n=0}^{+\infty}\left(\sum_{\nu=0}^{k-1} C_{n, 2 \nu} r^{2 \nu+1-n}\right) G_{n}(\zeta)+\sum_{n=0}^{+\infty}\left(\sum_{\nu=0}^{k-1} D_{n, 2 \nu} r^{2 \nu+1-n}\right) H_{n}(\zeta),
\end{aligned}
$$

where $A_{n, i}, B_{n, i}, C_{n, i}, D_{n, i}, n=0,1,2,3, \ldots, i=0,2,4, \ldots, 2 k-2$ are constants. 
Proof. From Theorem 1 and the theory of the generalized eigenfunctions we conclude that the derived eigenfunctions of Theorem 1 form a complete set of solutions, so Lemma 3 holds true.

\section{Conclusion}

In the present paper, we derived the 0-eigenspace of the operator $E^{2 k}, k \in \mathbb{N}^{*}$ and the corresponding solution of the equation $E^{2 k} \psi=0$ in the case of the spherical coordinate system. The eigenfunctions are products between appropriate powers of the Euclidean distance and Gegenbauer functions of both kinds. Moreover, we showed a solution for the equation $E^{2 k} \psi=0$, which proves that solution of $E^{2 k} \psi=0$ can be derived using only eigenfunctions of Stokes operator. This decomposition is not unique. However every solution of $E^{2 k} \psi=0$ can be decomposed as (6) indicates.

\section{References}

[1] P. Moon and D. E. Spencer, Field Theory Handbook, Springer-Verlag, 1961.

[2] P. M. Morse and H. Feshbach, Methods of Theoretical Physics, McGraw-Hill, 1953.

[3] R. P. Eisenhart, Separable systems of Stackel, Annals of Mathematics 35(2) (1934), 284-305.

DOI: https://doi.org/10.2307/1968433

[4] P. Moon and D. E. Spencer, Separability conditions for the Laplace and Helmholtz equations, Journal of the Franklin Institute 253(6) (1952), 585-600.

DOI: https://doi.org/10.1016/0016-0032(52)90682-0

[5] P. Moon and D. E. Spencer, Separability in a class of coordinate systems, Journal of the Franklin Institute 254(3) (1952), 227-242.

DOI: https://doi.org/10.1016/0016-0032(52)90460-2

[6] P. Moon and D. E. Spencer, Some coordinate systems associated with elliptic functions, Journal of the Franklin Institute 255(6) (1953), 531-543.

DOI: https://doi.org/10.1016/0016-0032(53)90302-0

[7] P. Moon and D. E. Spencer, Theorems on separability in Riemannian $n$-space, Proceedings of the American Mathematical Society 3(4) (1952), 635-642.

DOI: https://doi.org/10.1090/S0002-9939-1952-0049439-7 
[8] P. Moon and D. E. Spencer, Recent investigations of the separation of Laplace's equation, Proceedings of the American Mathematical Society 4(2) (1953), 302-307.

DOI: https://doi.org/10.1090/S0002-9939-1953-0053335-X

[9] E. Almansi, Sull'integrazione dell'equazione differenziale $\Delta^{2 n}=0$, Annali di Matematica Pura ed Applicata 2(1) (1899), 1-51.

DOI: https://doi.org/10.1007/BF02419286

[10] J. Happel and H. Brenner, Low Reynolds Number Hydrodynamics, Kluwer Academic Publishers, Dordrecht, The Netherlands, 1991.

[11] E. Protopapas, On the solution of irrotational Stokes flow in rotational system of coordinates, Submitted for publication.

[12] G. Dassios, M. Hadjinicolaou and A. C. Payatakes, Generalized eigenfunctions and complete semiseparable solutions for Stokes flow in spheroidal coordinates, Quarterly of Applied Mathematics 52(1) (1994), 157-191.

DOI: https://doi.org/10.1090/qam/1262325

[13] M. Hadjinicolaou and E. Protopapas, On the R-semiseparation of the Stokes bi-stream operator in inverted prolate spheroidal geometry, Mathematical Methods in the Applied Sciences 37(2) (2014), 207-211.

DOI: https://doi.org/10.1002/mma.2841

[14] M. Hadjinicolaou and E. Protopapas, Spectral decomposition of the Stokes flow operators in the inverted prolate spheroidal coordinates, IMA Journal of Applied Mathematics 80(5) (2015), 1475-1491.

DOI: https://doi.org/10.1093/imamat/hxv003

[15] M. Hadjinicolaou and E. Protopapas, Eigenfunction expansions for the Stokes flow operators in the inverted oblate coordinate system, Mathematical Problems in Engineering (2016); Article ID 9049131.

DOI: http://dx.doi.org/10.1155/2016/9049131

[16] S. Deo and A. Tiwari, On the solution of a partial differential equation representing irrotational flow in bispherical polar coordinates, Applied Mathematics and Computation 205(1) (2008), 475-477.

DOI: https://doi.org/10.1016/j.amc.2008.08.023

[17] M. Hadjinicolaou and E. Protopapas, Necessary and sufficient conditions for the separability and the R-separability of the irrotational Stokes equation, Submitted for publication.

[18] A. Charalambopoulos and G. Dassios, Complete decomposition of axisymmetric Stokes flow, International Journal of Engineering Science 40(10) (2002), 1099-1111.

DOI: https://doi.org/10.1016/S0020-7225(02)00004-6

[19] G. Dassios and P. Vafeas, On the spheroidal semiseparation for Stokes flow (2008); Article ID 135289.

DOI: https://doi.org/10.1155/2008/135289

[20] N. N. Lebedev, Special Functions and their Applications, Dover Publications, 1972. 